\title{
Unusual gastrointestinal and cutaneous toxicities by bleomycin, etoposide, and cisplatin: a case report with pharmacogenetic analysis to personalize treatment
}

\author{
Marzia Del $\operatorname{Re}^{1} \cdot$ Tiziana Latiano $^{2} \cdot$ Leonardo Fidilio $^{1} \cdot$ Giuliana Restante $^{1}$. \\ Franco Morelli $^{2} \cdot$ Evaristo Maiello $^{2} \cdot$ Romano Danesi $^{1}$
}

Received: 8 December 2016 / Accepted: 24 January 2017 /Published online: 7 March 2017

(C) European Association for Predictive, Preventive and Personalised Medicine (EPMA) 2017

\begin{abstract}
The standard treatment of testicular germ cell tumors is based on the combination of bleomycin, etoposide, and cisplatin (PEB). However, this treatment may be associated with severe adverse reactions, such as hematological and non-hematological toxicities. Here, we report a case of a patient suffering from severe PEB-related toxicities, to whom pharmacogenetic analyses were performed, comprising a panel of genes involved in PEB metabolism. The analysis revealed the presence of a complex pattern of polymorphisms in GSTP1, UGT1A1 (TA)6/7, UGT1A7, and ABCB1. The present case shows that a pharmacogenetic approach can help in the management of adverse drug reactions in order to predict, prevent, and personalize treatments.
\end{abstract}

Keywords Pharmacogenetics · Toxicity ·

Bleomycin-etoposide-cisplatin combined treatment .

Testicular germ cell cancer $\cdot$ Polymorphisms $\cdot$ Predictive

preventive personalized medicine $\cdot$ Precision medicine

\section{Introduction}

Germ cell tumors (GCT) are a group of heterogeneous neoplasms most frequently occurring in testes and ovaries. Type II

Marzia Del Re and Tiziana Latiano are co-first authors of the manuscript

Romano Danesi

romano.danesi@unipi.it

1 Clinical Pharmacology and Pharmacogenetics Unit, Department of Clinical and Experimental Medicine, University of Pisa, 55, Via Roma, 56126 Pisa, Italy

2 Medical Oncology Unit, Casa Sollievo della Sofferenza Hospital, San Giovanni Rotondo, Foggia, Italy
GCT, the most common form, is formed by seminomas and non-seminomas, with an incidence of about $6 / 100,000$ per year [1]. Up to $50 \%$ of all testicular GCT (TGCT) are seminomas, with a median age at diagnosis of 35 years. Non-seminomas usually develop in $30 \%$ of cases, most commonly in 25-year-old patients. The remaining $20 \%$ are characterized by combined seminomas and non-seminomas [2]. TGCT represents the prevalent malignancy in 20 - and 40 year-old caucasian males and include almost $60 \%$ of solid cancers in young men [3]. Despite the incidence of TGCT increased by $70 \%$ during the last 20 years [1], TGCT is among the most curable solid tumors with 10-year survival rate of more than $95 \%$. The standard treatment of TGCT is the chemotherapeutic combination of bleomycin, etoposide, and cisplatin (PEB) [4], sometimes associated with severe adverse reactions, such as hematological (leukopenia grades 3 and 4, anemia, thrombocytopenia, and neutropenic fever) and nonhematological toxicities (vomiting, diarrhea, and stomatitis) [5].

Cisplatin-based chemotherapy is the cornerstone in the management of metastatic TGCT and the PEB regimen is usually well tolerated with manageable acute and chronic toxicities including neutropenia, anemia — not usually requiring transfusions - and thrombocytopenia. Neutropenic fever occurs in approximately $10-15 \%$ of patients receiving etoposide and cisplatin and it is more common with the addition of bleomycin [5].

Here, we report an uncommon case of severe toxicity in a patient treated by PEB regimen.

A 45-year-old man affected by advanced seminoma was treated with only one course of PEB chemotherapy because grade 4 neutropenic fever, mucositis, and intestinal obstruction occurred on day 9 after the beginning of chemotherapy. Abdominal X-ray showed dilated bowel loops and multiple air-fluid levels. The patient was hospitalized and given 
infusional antibiotics, parenteral nutrition, and hemopoietic growth factors (G-CSF). In the following days, red blood cell and platelet transfusions were needed due to severe anemia and thrombocytopenia. On day 23 rd from the beginning of chemotherapy, the patient underwent esophagogastroduodenoscopy (EGD) for the persistence of anemia and dysphagia; a mucositis likely due to Candida albicans was detected (Fig. 1). On day 26th, he showed symptoms of digestive bleeding (hematemesis, melena, rectal bleeding) and underwent selective arteriography that, however, did not allow the identification of the site of bleeding. A subsequent EGD demonstrated duodenal and gastric ulcers as well as erosive gastro-duodenitis. In the following days the patient was again treated with transfusions of blood, platelets and plasma and tranexamic acid was added for the persistence of melena. In this occasion another endoscopic examination demonstrated an additional erosive duodenojejunitis with oozing bleeding. The patient also presented skin toxicity similar to that induced by capecitabine (Fig. 2). He was finally discharged in good general medical condition on day 49th from the beginning of the chemotherapy. Since the inactivating metabolic pathways of PEB regimen involve the glutathione-S-transferase (GST) and uridine diphosphateglucuronosyltransferase (UGT) enzymes (in particular for cisplatin and etoposide), and renal excretion is mediated by the ATP-binding cassette (ABC) transporters, we performed a pharmacogenetic analysis to explain the reported toxicity (Fig. 3).

\section{Materials and methods}

In order to investigate the genetic basis of severe toxicity, the following polymorphisms of genes involved in PEB metabolism were examined: glutathione-S-transferase isoform $1 \mathrm{P}$ (GSTP1) c.313A $>$ G; uridine diphosphateglucuronosyltransferase isoforms 1A1 (UGT1A1) (TA)6/7

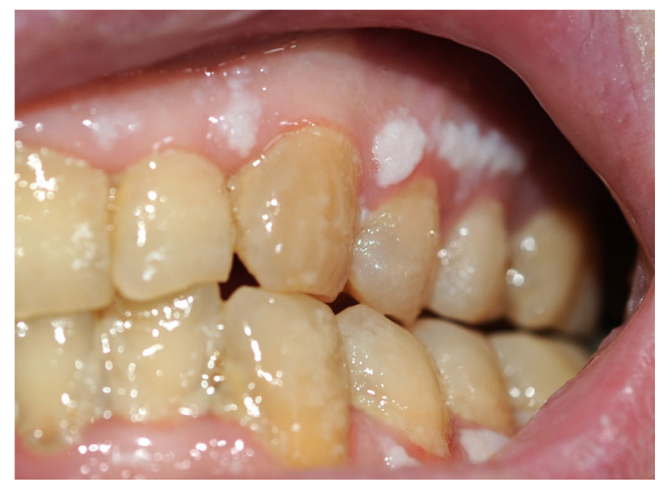

Fig. 1 Severe mucositis in this patient receiving PEB. The figure shows the presence of whitish patches on the surface of gums due to the presence of underlying sores, as well as red, shiny, and swollen gums associated with poor oral hygiene (diffuse dental plaques are visible)

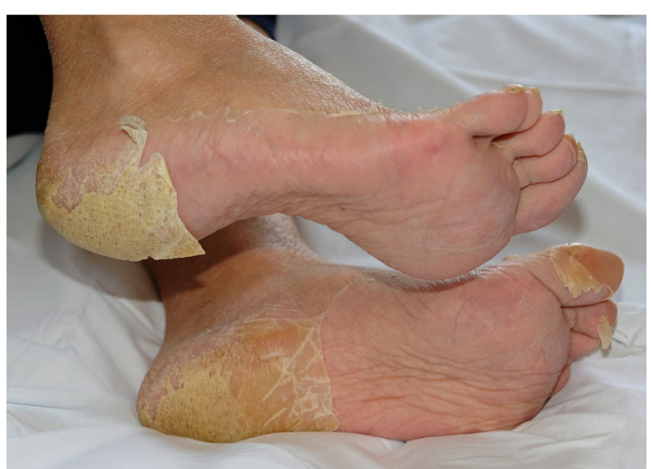

Fig. 2 Severe hand-foot syndrome in this patient receiving PEB. The figure shows the presence of cracked and peeling skin of the feet, patchy redness, and calluses of the soles. The skin shows tightness and thinning

and c. $-3156 \mathrm{G}>\mathrm{A}$, UGT1A7 c. $387 \mathrm{~T}>\mathrm{G} / 391 \mathrm{C}>\mathrm{A} / 392 \mathrm{G}>\mathrm{A}$, and c. $-57 \mathrm{~T}>\mathrm{G}$; ATP-binding cassette $\mathrm{B} 1$ (ABCB1) c. $1236 \mathrm{~T}>\mathrm{C}, \mathrm{c} .2677 \mathrm{~T}>\mathrm{G}$ and c.3435C $>\mathrm{T}$. These variants were selected because they are associated with a reduced metabolism of cisplatin and etoposide by GSTP1 and of etoposide by UGT and impaired renal transport and excretion of etoposide by $\mathrm{ABCB} 1$ (frequency of selected variants are reported in Table 1). DNA was extracted from peripheral blood using the BioRobot EZ1 (Qiagen $®$, Valencia, CA, USA) and mutations were analyzed by automatic sequencing on ABI 3100 automated DNA sequencer (Applied Biosystems ${ }^{\circledR}$, Foster City, CA).

\section{Results}

Sequence analysis revealed the presence of a complex pattern of polymorphisms: GSTP1 c.313AG; UGT1A1 (TA)6/7 and c.-3156GA; UGT1A7 c.-57TG and c.387GG/391AA/392AA, and ABCB1 c.2677TG, c.3435CT and c.1236CC. Taken together, the genetic profile could be associated with an impaired hepatic and renal clearance, thus explaining the accumulation of active drugs and the observed toxicity.

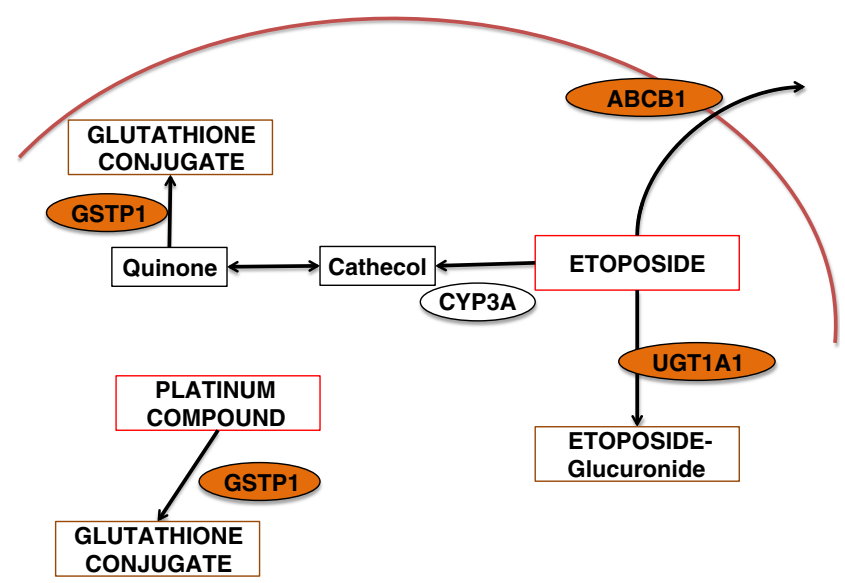

Fig. 3 Enzymes involved in the metabolic pathway of PEB regimen 
Table 1 Genotype frequencies of analyzed variants (https://www. ncbi.nlm.nih.gov/snp)

\begin{tabular}{|c|c|c|}
\hline Gene & Polymorphism & Genotype frequencies \\
\hline GSTP1 & c. $313 \mathrm{~A}>\mathrm{G}$ & AA $32 \%-$ AG $55 \%-$ GG $13 \%$ \\
\hline \multirow[t]{2}{*}{ UGT1A1 } & (TA) $6 / 7$ & $6 / 644 \%-6 / 747 \%-7 / 79 \%$ \\
\hline & c. $-3156 \mathrm{G}>\mathrm{A}$ & GG $5 \%-$ GA $43 \%-$ AA $52 \%$ \\
\hline UGT1A7 & $\begin{array}{l}\text { c. } 387 \mathrm{~T}>\mathrm{G} / \mathrm{c} .391 \mathrm{C}>\mathrm{A} / \mathrm{c} .392 \mathrm{G}>\mathrm{A} \\
\text { c. }-57 \mathrm{~T}>\mathrm{G}\end{array}$ & $\begin{array}{l}\text { TT/CC/GG } 29 \%-\text { TG/CA/AA } 47 \%-\text { GG/AA/AA } 22 \% \\
\text { TT } 34 \% \text { - TG } 53 \%-\text { GG } 12 \%\end{array}$ \\
\hline \multirow[t]{3}{*}{ ABCB1 } & c. $1236 \mathrm{~T}>\mathrm{C}$ & TT $76 \%-$ CT $57 \%-$ CC $26 \%$ \\
\hline & c. $2677 \mathrm{~T}>\mathrm{G}$ & TT19\% - TG55\% - GG26\% \\
\hline & c. $3435 \mathrm{C}>\mathrm{T}$ & CC $15 \%$ - CT $55 \%$ - TT $29 \%$ \\
\hline
\end{tabular}

\section{Discussion}

The present case report describes the role of deficient variants of enzymes of PEB metabolic pathway as a possible cause of toxicity and highlights the deleterious effect of their combination on treatment tolerability. In particular, bleomycin is mainly excreted by the kidneys and inactivated by bleomycin hydrolase (BMH). In particular, the c.1450A $>$ G polymorphism in the BMH gene results in a reduced enzymatic activity and possibly development of pulmonary toxicity [6]. However, not enough evidences substantiate this correlation.

The metabolic pathway of cisplatin involves the GST enzymes, isoforms T1 and P1 (GSTT1, GSTP1) [7]. GSTs are a family of eukaryotic and prokaryotic phase II metabolic isozymes regulating the conversion of toxic compounds to hydrophilic metabolites during detoxification process. They have an important role in the accumulation of DNA adducts and oxidative DNA damage when their activity is reduced [8]. Seven classes of cytosolic GSTs have been discovered [9] and are implicated in the metabolism and resistance to several drugs, including cisplatin [10]. The main human GST isoform is the P1 (GSTP1), localized in many tissues, including erythrocytes, placenta, lung, brain, muscle, and liver [11]. The prevalent functional polymorphism of GSTP1 is an isoleucine/valine change at codon 105 (p.Ile105Val; c.313A $>\mathrm{G}$; rs1695). In a retrospective study, it has been shown that this polymorphism has a considerable impact on treatment tolerability in testicular cancer: patients administered with cisplatin-based chemotherapy carrying the c.313AA or c.313AG genotype had more than three-fold increased risk of severe numbness and paresthesia in bilateral toes, Raynaud-like phenomena, tinnitus and hearing impairment than those with the c.313GG genotype [12].

Etoposide is mainly metabolized by cytochromes P-450 isoforms $3 \mathrm{~A} 4$ and $3 \mathrm{~A} 5$ (CYP3A4, CPY3A5) into the active catechol metabolite, a topoisomerase inhibitor [13, 14], while GSTT1 and P1 and UGT1A1 inactivate the drug [15-18]. UGTs are a large family of enzymes catalyzing the addition of a glucuronosyl group to small hydrophobic molecules to generate more polar compounds that may be easily cleared from the body. Moreover, the UGT family plays an important role in the detoxification processes of endogenous and exogenous products (bile acids, dietary amines and flavones, phenols, steroids, bilirubin) [19]. According to Mackenzie et al. [20], 18 UGT-isoforms have been recognized in humans, most of them located in the liver. To date, 113 UGT1A different variants have been described having a notable significance in pharmacogenetic research [21]. The most clinically relevant variant affects the number of TA dinucleotides in the TATA box region of UGT1A1 [22], from 6 [A(TA)6TAA] (wild type) to 7 TA repeats [A(TA)7TAA] (UGT1A1*28) [23, 24]. While UGT1A1 is primarily responsible for conjugation of bilirubin, the presence of an additional TA repeat in the TATA region of the UGT1A1 promoter decreases UGT1A1 production, leading to a reduced glucuronidation, consistent with a diagnosis of Gilbert's syndrome [25, 26]. UGT1A1 also catalyzes the glucuronidation of several drugs, including etoposide, and for this reason patients with a decresed enzyme activity have a higher risk of developing toxicities when treated with UGT substrates [17]. In addition to the UGT1A1*28, other polymorphisms have been identified in the UGT gene. The UGT1A1*93 (c.-3156G>A; rs10929302) is located in the promoter region and decreases the UGT1A1 protein levels [27]. UGT1A1*93 is in partial linkage disequilibrium with the UGT1A1*28 [28] and may be a more robust biomarker for neutropenia than UGT1A1*28 [27]. The activity of UGT1A7 may be reduced by the presence of c.-57T $>\mathrm{G}$ in the promoter region, which is correlated with a $70 \%$ reduction of the gene transcription [29] while others may be linked to severe hematologic toxicity of irinotecan, i.e., c.387T $>\mathrm{G}$, c. $391 \mathrm{C}>\mathrm{A}, \mathrm{c} .392 \mathrm{G}>\mathrm{A}$, and c.622T>C [28, 30, 31].

The efflux of conjugated or unconjugated metabolites of etoposide is mediated by $\mathrm{ABCB} 1, \mathrm{ABCC} 1$, and $\mathrm{ABCC} 3[32$, 33]. The $\mathrm{ABCs}$ are transmembrane proteins that use energy from ATP to transport various molecules through the cell membranes (34). ABCB1 is able to transfer hydrophobic substrates, including colchicine, etoposide, doxorubicin, and vinblastine [34]. ABCB1 is expressed in many secretory cells including kidney, liver, intestine, and adrenal glands. The main polymorphisms of $\mathrm{ABCB} 1$ are frequently in linkage 
disequilibrium and include c.1236T $>\mathrm{C}$ (rs1128503, p.Gly412Gly), c.2677T>G/A (rs2032582, p.Ser893Ala/Thr), and c.3435>C (rs1045642, p.Ile1145Ile) [35]. The c.3435C >T affects $\mathrm{ABCB} 1 \mathrm{mRNA}$ levels and protein expression, whereas the non-synonymous c. $2677 \mathrm{G}>\mathrm{T}$ has been reported to increase transport activity [36]; finally, the c.1236T $>$ C seems to be correlated to increased drug exposure [37]. The patient was bearing the following variants (ABCB1 c.2677TG, c. $3435 \mathrm{CT}$, and c.1236CC) that are potentially associated with impaired clearance and increased drug levels.

In conclusion, the severe toxicities developed in this patient could be associated with an impaired clearance activity of GST, UGT, and ABC. The results of the present work demonstrate that pharmacogenetic analysis of metabolic pathways of drugs may help identify patients at risk of adverse events, allowing a safer and personalized approach to chemotherapy [38]. Unfortunately, there are no widely accepted guidelines for pharmacogenetic screening, except for some clinical relevant situation. Therefore, further investigations in this field are needed to clarify the complexity of drug metabolism and identify patients at high risk.

\section{Conclusions and expert recommendations}

The present case highlights the importance of including pharmacogenetic tests in the clinical practice. The drug metabolism can be altered by genetic variants that may result in variations of enzyme activity. Consequently, individuals classified as "slow metabolizers" may have high concentrations of drugs and increased risk of toxicity, and "fast metabolizers" may be undertreated in certain cases. In this regard, pharmacogenetic tests may help in the selection of drugs and their dosages reducing the risk of toxicities. Nowadays, a treatment adaptation based on pharmacogenetic results is often required (i.e., fluoropyrimidines and DPD, irinotecan and UGT, warfarin and CYP2C9 and VKORC1) [39]. Due to an expansion of the pharmacogenetic tests, costs have become very low, also with the introduction of new high throughput genotyping techniques. Of course, the use of pharmacogenetic test in clinical practice has still to be regulated, and specific guidelines are even more required.

Acknowledgements No financial interests to disclose.

\section{References}

1. Elzinga-Tinke JE, Dohle GR, Looijenga LH. Etiology and early pathogenesis of malignant testicular germ cell tumors: towards possibilities for preinvasive diagnosis. Asian J Androl. 2015;17(3): 381-93.
2. Oosterhuis JW, Looijenga LH. Testicular germ-cell tumours in a broader perspective. Nat Rev Cancer. 2005;5(3):210-22.

3. Looijenga LH. Human testicular (non)seminomatous germ cell tumours: the clinical implications of recent pathobiological insights. J Pathol. 2009;218(2):146-62.

4. Williams SD, Birch R, Einhorn LH, Irwin L, Greco FA, Loehrer PJ. Treatment of disseminated germ-cell tumors with cisplatin, bleomycin, and either vinblastine or etoposide. N Engl J Med. 1987;316(23):1435-40.

5. Kawai K, Ando S, Hinotsu S, et al. Completion and toxicity of induction chemotherapy for metastatic testicular cancer: an updated evaluation of Japanese patients. Jpn J Clin Oncol. 2006;36(7):425-31.

6. Nuver J, Lutke Holzik MF, Van Zweeden M, et al. Genetic variation in the bleomycin hydrolase gene and bleomycin-induced pulmonary toxicity in germ cell cancer patients. Pharmacogenet Genomics. 2005;15(6):399-405.

7. Pereira D, Assis J, Gomes M, Nogueira A, Medeiros R. Improvement of a predictive model in ovarian cancer patients submitted to platinum-based chemotherapy: implications of a GST activity profile. Eur J Clin Pharmacol. 2016;72(5):545-53.

8. Wu K, Wang X, Xie Z, Liu Z, Lu Y. Glutathione S-transferase P1 gene polymorphism and bladder cancer susceptibility: an updated analysis. Mol Biol Rep. 2013;40(1):687-95.

9. Sheehan D, Meade G, Foley VM, Dowd CA. Structure, function and evolution of glutathione transferases: implications for classification of non-mammalian members of an ancient enzyme superfamily. Biochem J. 2001;360(Pt 1):1-16.

10. Klys HS, Whillis D, Howard G, Harrison DJ. Glutathione Stransferase expression in the human testis and testicular germ cell neoplasia. Br J Cancer. 1992;66(3):589-93.

11. Goodrich JM, Basu N. Variants of glutathione s-transferase pi 1 exhibit differential enzymatic activity and inhibition by heavy metals. Toxicol In Vitro. 2012;26(4):630-5.

12. Oldenburg J, Kraggerud SM, Brydoy M, Cvancarova M, Lothe RA, Fossa SD. Association between long-term neuro-toxicities in testicular cancer survivors and polymorphisms in glutathione-stransferase-P1 and -M1, a retrospective cross sectional study. J Transl Med. 2007;5:70.

13. Relling MV, Nemec J, Schuetz EG, Schuetz JD, Gonzalez FJ, Korzekwa KR. O-demethylation of epipodophyllotoxins is catalyzed by human cytochrome P450 3A4. Mol Pharmacol. 1994;45(2):352-8.

14. Zhuo X, Zheng N, Felix CA, Blair IA. Kinetics and regulation of cytochrome P450-mediated etoposide metabolism. Drug Metab Dispos. 2004;32(9):993-1000.

15. Mans DR, Lafleur MV, Westmijze EJ, et al. Reactions of glutathione with the catechol, the ortho-quinone and the semi-quinone free radical of etoposide. Consequences for DNA inactivation. Biochem Pharmacol. 1992;43(8):1761-8.

16. Hande K, Bennett R, Hamilton R, Grote T, Branch R. Metabolism and excretion of etoposide in isolated, perfused rat liver models. Cancer Res. 1988;48(20):5692-5.

17. Wen Z, Tallman MN, Ali SY, Smith PC. UDPglucuronosyltransferase $1 \mathrm{~A} 1$ is the principal enzyme responsible for etoposide glucuronidation in human liver and intestinal microsomes: structural characterization of phenolic and alcoholic glucuronides of etoposide and estimation of enzyme kinetics. Drug Metab Dispos. 2007;35(3):371-80.

18. Naritomi Y, Terashita S, Kagayama A, Sugiyama Y. Utility of hepatocytes in predicting drug metabolism: comparison of hepatic intrinsic clearance in rats and humans in vivo and in vitro. Drug Metab Dispos. 2003;31(5):580-8.

19. Meech R, Mackenzie PI. Structure and function of uridine diphosphate glucuronosyltransferases. Clin Exp Pharmacol Physiol. 1997;24(12):907-15. 
20. Mackenzie PI, Owens IS, Burchell B, et al. The UDP glycosyltransferase gene superfamily: recommended nomenclature update based on evolutionary divergence. Pharmacogenetics. 1997;7(4):255-69.

21. Barbarino JM, Haidar CE, Klein TE, Altman RB. PharmGKB summary: very important pharmacogene information for UGT1A1. Pharmacogenet Genomics. 2014;24(3):177-83.

22. Innocenti F, Grimsley C, Das S, et al. Haplotype structure of the UDP-glucuronosyltransferase $1 \mathrm{~A} 1$ promoter in different ethnic groups. Pharmacogenetics. 2002;12(9):725-33.

23. Bosma PJ, Chowdhury JR, Bakker C, et al. The genetic basis of the reduced expression of bilirubin UDP-glucuronosyltransferase 1 in Gilbert's syndrome. N Engl J Med. 1995;333(18):1171-5.

24. Beutler E, Gelbart T, Demina A. Racial variability in the UDPglucuronosyltransferase 1 (UGT1A1) promoter: a balanced polymorphism for regulation of bilirubin metabolism? Proc Natl Acad Sci U S A. 1998;95(14):8170-4.

25. Iyer $\mathrm{L}$, King $\mathrm{CD}$, Whitington $\mathrm{PF}$, et al. Genetic predisposition to the metabolism of irinotecan (CPT-11). Role of uridine diphosphate glucuronosyltransferase isoform 1A1 in the glucuronidation of its active metabolite (SN-38) in human liver microsomes. J Clin Invest. 1998;101(4):847-54.

26. Iyer L, Das S, Janisch L, et al. UGT1A1*28 polymorphism as a determinant of irinotecan disposition and toxicity. Pharmacogenomics J. 2002;2(1):43-7.

27. Crona DJ, Ramirez J, Qiao W, et al. Clinical validity of new genetic biomarkers of irinotecan neutropenia: an independent replication study. Pharmacogenomics J. 2016;16(1):54-9.

28. Cecchin E, Innocenti F, D'andrea M, et al. Predictive role of the UGT1A1, UGT1A7, and UGT1A9 genetic variants and their haplotypes on the outcome of metastatic colorectal cancer patients treated with fluorouracil, leucovorin, and irinotecan. J Clin Oncol. 2009;27(15):2457-65.

29. Fujita K, Ando Y, Nagashima F, et al. Novel single nucleotide polymorphism of UGT1A7 gene in Japanese. Drug Metab Pharmacokinet. 2006;21(1):75-8.
30. Huang MJ, Yang SS, Lin MS, Huang CS. Polymorphisms of uridine-diphosphoglucuronosyltransferase $1 \mathrm{~A} 7$ gene in Taiwan Chinese. World J Gastroenterol. 2005;11(6):797-802.

31. Kong SY, Ki CS, Yoo BC, Kim JW. UGT1A7 haplotype is associated with an increased risk of hepatocellular carcinoma in hepatitis B carriers. Cancer Sci. 2008;99(2):340-4.

32. Jedlitschky G, Leier I, Buchholz U, Barnouin K, Kurz G, Keppler D. Transport of glutathione, glucuronate, and sulfate conjugates by the MRP gene-encoded conjugate export pump. Cancer Res. 1996;56(5):988-94.

33. Zelcer N, Saeki T, Reid G, Beijnen JH, Borst P. Characterization of drug transport by the human multidrug resistance protein 3 (ABCC3). J Biol Chem. 2001;276(49):46400-7.

34. Dean M, Rzhetsky A, Allikmets R. The human ATP-binding cassette (ABC) transporter superfamily. Genome Res. 2001;11(7): $1156-66$.

35. Kroetz DL, Pauli-Magnus C, Hodges LM, et al. Sequence diversity and haplotype structure in the human ABCB1 (MDR1, multidrug resistance transporter) gene. Pharmacogenetics. 2003;13(8):48194.

36. Wang D, Johnson AD, Papp AC, Kroetz DL, Sadee W. Multidrug resistance polypeptide 1 (MDR1, ABCB1) variant $3435 \mathrm{C}>\mathrm{T}$ affects mRNA stability. Pharmacogenet Genomics. 2005;15(10): 693-704.

37. Hodges LM, Markova SM, Chinn LW, et al. Very important pharmacogene summary: ABCB1 (MDR1, P-glycoprotein). Pharmacogenet Genomics. 2011;21(3):152-61.

38. Golubnitschaja $\mathrm{O}, \mathrm{Baban} \mathrm{B}$, Boniolo G, et al. Medicine in the early twenty-first century: paradigm and anticipation - EPMA position paper 2016. EPMA J. 2016;7:23.

39. Grech $\mathrm{G}$, Zhan $\mathrm{X}$, Yoo BC, et al. EPMA position paper in cancer: current overview and future perspectives. EPMA J. 2015;6(1):9. 